\title{
EFECTO AGUDO DE LOS ENTRENAMIENTOS DE FUERZA, VELOCIDAD, PLIOMETRÍA Y VELOCIDAD CONTRA RESISTENCIA EN LA CARRERA DE VELOCIDAD
}

\section{ACUTE EFFECT OF STRENGTH, SPEED, PLYOMETRIC AND RESISTED SPEED TRAININGS ON RUNNING SPEED PERFORMANCE}

\author{
José Francisco Barquero Jiménez ${ }^{1}$ y Walter Salazar Rojas ${ }^{2}$ \\ ${ }^{1}$ Posgrado en Ciencias del Movimiento Humano, Universidad de Costa Rica, Costa Rica. \\ ${ }^{2}$ Escuela de Educación Física y Deportes, Universidad de Costa Rica, Costa Rica. \\ jose.fbju@gmail.com; walter.salazar@ucr.ac.cr \\ Envío original: 2020-01-28 Reenviado: 2020-04-16, 2020-05-18 Aceptado: 2020-05-19 \\ Publicado: 2020-07-01
}

Doi: https://doi.org/10.15517/pensarmov.v18i2.40315

\begin{abstract}
RESUMEN
La velocidad juega un papel fundamental en el rendimiento deportivo tanto en deportes individuales como colectivos. Los objetivos de este estudio son: a) determinar el efecto agudo de los tipos de entrenamiento de Fuerza, Velocidad, Pliometría y Velocidad contra Resistencia en la carrera de velocidad y sus fases, y b) realizar el análisis del efecto agudo de estos tipos de entrenamiento de manera individual. Once velocistas (edad promedio de 21.8 años \pm 3.04 , talla de $1.764 \mathrm{~m} \pm 0.062$ y peso de $69.645 \mathrm{~kg} \pm 4.946$ ), con experiencia como velocistas realizaron cinco tratamientos agudos ( $F, V, P, V R$ y control), para los cuales se aleatorizó el orden. Para cada tratamiento, realizaron un pre test y un pos test de 100 metros con mediciones de intervalos cada 20 metros. Se realizaron 2 análisis de varianza (ANOVA) de medidas repetidas de 2 y 3 vías, con el objetivo de analizar el efecto de los tratamientos en el tiempo total de carrera y en cada uno de los intervalos de la carrera de velocidad respectivamente. También, se realizó un análisis individual, utilizando estadísticos descriptivos y gráficos. No hubo interacción significativa entre las mediciones y los tipos de entrenamiento ( $F=1.733 p=0.162)$, ni entre las mediciones, los tipos de entrenamiento y los intervalos de distancia $(F=0.569 p=0.903)$. En el análisis
\end{abstract}


individual, se observa como los cambios en la velocidad según el tipo de tratamiento varían entre sujetos, siendo el tratamiento de velocidad con el que más sujetos mejoran.

Palabras Clave: velocidad, fuerza, velocidad contra resistencia, pliometría y efecto agudo.

\begin{abstract}
Speed plays a main role in sport performance in both collective and individual sports. The aims of this study are: a) to determinate the acute effect of speed, strength, resisted speed and plyometric training on speed performance and speed phases, and b) to analyze the performance data with individual and group analysis. Eleven sprinters (mean age of $21.8 \pm 3.04$, height of $1.764 \mathrm{~m} \pm 0.062$ and weight of $69.645 \mathrm{~kg} \pm 4.946)$, with experience as sprinters in 100,200 or $400 \mathrm{~m}$ events participated in 5 training sessions assigned in a random order. Each condition was performance on different days, separated by at least 48 hours, and a 100-meter test was applied before and after each condition. Time recording was performance every 20 meters in the $100 \mathrm{~m}$ test. Two way and three-way variance analysis (ANOVA) of repeated measurements were performance with the aim of studied the effect of the types of training on the speed race and on their distance intervals. Statistical descriptive and graphics were used for the individual analysis. For the group analysis, no significant interaction was found between measurements and types of trainings $(F=1.733 p=0.162)$, and between measurements, distance intervals and types of trainings $(F=0.569 p=0.903)$. However, in the individual analysis it is noted the differences between athletes on the response to each training type, being the speed training the one in which more subjects enhanced.
\end{abstract}

Key words: speed, sprint, strength, resisted sprint, sled sprint, plyometric and acute effect. 


\section{INTRODUCCIÓN}

El entrenamiento de la velocidad es esencial en la mayoría de deportes colectivos o individuales (Van Den Tillaar, Teixeira y Marinho, 2017), destacando en las pruebas de velocidad del atletismo debido a que son mínimas las diferencias (1, 2 o 3 centésimas de segundo) que permiten triunfar en una prueba de 100 o 200 metros.

Debido a la importancia de la velocidad en el rendimiento deportivo y de su mejora para entrenadores y atletas, se han investigado diferentes tipos de entrenamiento con los que se pueden mejorar la velocidad, destacando los entrenamientos de fuerza, velocidad, pliometría y velocidad contra resistencia (Barquero y Salazar, 2018; Centeno y Salazar, 2017; Sáez, Requena y Cronin, 2012).

Se conoce de estudios meta-analíticos (Barquero y Salazar, 2018; Centeno y Salazar, 2017; Sáez et al., 2012), que los entrenamientos de fuerza, velocidad, pliometría y velocidad contra resistencia dan como resultado una mejora en la carrera de velocidad $(T E=0.55$; $T E=$ 0.50-0.40; $T E=0.41-0.37 ; T E=0.36$, respectivamente).

Sin embargo, estos efectos positivos conocidos de los tipos de entrenamiento mencionados en la carrera de velocidad, son producto de estudios de efecto crónico, existiendo poca investigación sobre el efecto agudo de estos en la carrera de velocidad.

Es importante aclarar antes de pasar a describir lo que se conoce del efecto agudo de cada tipo de entrenamiento en la carrera de velocidad, que, en la literatura científica se pueden localizar estudios de efecto agudo, con este nombre o con los términos de potenciación posactivación o de calentamiento. Incluyendo los tres términos una amplia variedad de estudios con características similares, donde la mayor diferencia está en que muchos estudios de calentamiento o potenciación pos activación no se pueden meta-analizar debido a la falta de aplicación de un pre-test.

Los estudios sobre el efecto agudo del entrenamiento de fuerza en la carrera de velocidad presentan resultados diversos, destacando estudios con efectos positivos (Chatzopoulos et al., 2007; Matthews, Matthews y Snook, 2004; Mcbride, Nimphius y Erickson, 2005; Rahman, 2007; Seitz, Trajano y Haff., 2014; Yetter y Moir, 2008) y estudios sin cambios en la velocidad (Bevan et al., 2010; Crewther et al., 2011; Guggenheimer, Dickin, Reyes y Dolny, 2009; Lim y Kong, 2013).

Por su parte, los estudios del efecto agudo del entrenamiento de velocidad contra resistencia presentan resultados similares a los estudios de fuerza, destacando que el volumen de investigaciones es mucho menor, con dos investigaciones que muestran cambios positivos 
en la velocidad (Smith et al., 2014; Winwood, Posthumus, Cronin y Keogh, 2016) y dos sin cambios (Van Den Tillaar et al., 2017; Whelan, O'regan y Harrison., 2014).

Se localizaron solamente dos investigaciones que estudiaban el efecto agudo del entrenamiento de pliometría en la velocidad (Bomfim-Lima et al., 2011; Turner, Bellhouse, Kilduff y Russell, 2015), obteniendo las dos una mejora en el tiempo de carrera. Por su parte, de manera sorprendente no se localizó ninguna investigación que estudie el efecto agudo del entrenamiento de velocidad en la carrera de velocidad.

Además, una carencia con que cuenta la investigación tanto del efecto agudo como crónico de los tipos de entrenamiento, es que la mayoría de los estudios han investigado el efecto de los tipos de entrenamiento en el tiempo total en una o dos distancias (Kale y Acikada, 2016; Rumpf et al., 2014), faltando investigar si el efecto global observado en la velocidad es el mismo en cada fase de la carrera de velocidad.

Lo antepuesto debido a que, la carrera de velocidad cuenta con cuatro fases, siendo estas: la reacción, la aceleración, la velocidad máxima y la resistencia a la velocidad (Cetin, Hindistan y Ozkaya, 2018). El análisis de los efectos de los tipos de entrenamiento en cada una de las fases tiene igual importancia para los entrenadores que conocer el efecto global. Podría ser que uno de los métodos de entrenamiento sea más efectivo para desarrollar la velocidad en la fase de aceleración, mientras que otro método podría ser más efectivo para el desarrollo de la resistencia a la velocidad. De ser así, un entrenador podría combinar ambos métodos para obtener una mejoría global más significativa.

También, otra limitación de este campo de investigación, es la escasa cantidad de investigaciones que han estudiado el efecto de los tipos de entrenamiento en la velocidad en distancias mayores a los 40 metros y en velocistas (Haugen, Seiler, Sandbakk y Tønnessen, 2019), de forma que se pueda analizar su efecto en la resistencia a la velocidad o en la velocidad máxima para deportes individuales como el atletismo donde se conoce que el valor de velocidad máxima se alcanza entre las distancias de 60 y 80 metros y no en 40 metros o antes como en el caso de los deportes colectivos (Haugen et al., 2019; Slawinski et al., 2017). En la tabla 1, se reportan la cantidad de investigaciones que han estudiado el efecto agudo de alguno de los tipos de entrenamientos de fuerza, velocidad, velocidad contra resistencia y pliometría en la carrera de velocidad por cada intervalo de 20 metros, donde se puede apreciar la falta de estudios donde se evalúen las fases de velocidad máxima y resistencia a la velocidad. 
Tabla 1.

Cantidad de estudios de efecto agudo realizados por intervalo de distancia.

\begin{tabular}{cccccc}
\hline Intervalo de distancia & $0-20 m$ & $20-40 m$ & $40-60 m$ & $60-80 m$ & $80-100 m$ \\
\hline Cantidad de estudios & 11 & 7 & 2 & 0 & 0
\end{tabular}

Nota: $\mathrm{m}=$ metros. Fuente: elaboración propia.

Otro aspecto de gran importancia para los entrenadores, es conocer si el efecto de cada uno de los tipos de entrenamiento en la carrera de velocidad funciona igual en cada uno de sus atletas, razón por la que es relevante no solo analizar el efecto global de un tratamiento sino también el efecto en cada individuo. Este tipo de investigaciones no se han realizado en el área del entrenamiento de la velocidad, sin embargo, si se han realizado anteriormente en investigaciones del entrenamiento de fuerza y en estudios descriptivos sobre las características de la puesta en punta en deportes de resistencia (Barrantes Segura y Aragón Vargas, 2018; Tønnessen et al., 2014)

Por lo tanto, dada la necesidad de estudiar el efecto agudo de los tipos de entrenamiento de fuerza, velocidad, pliometría y velocidad contra resistencia en la carrera de velocidad, así como su respuesta en distancias mayores a los 40 metros y con tiempos parciales que permitan estudiar las fases de la carrera de la velocidad, los objetivos de esta investigación son: 1. Determinar el efecto agudo de los tipos de entrenamiento de fuerza, velocidad, pliometría y velocidad contra resistencia en la carrera de velocidad y sus fases. 2 . Realizar el análisis anterior de manera grupal e individual.

La importancia de esta investigación, recalca en la necesidad por parte de los atletas y entrenadores en conocer el efecto agudo de los tipos de entrenamiento de fuerza, velocidad, pliometría y velocidad contra resistencia en la carrera de velocidad y sus fases. Lo anterior, permite valorar la utilización de los tipos de entrenamiento como calentamiento previo a sus entrenamientos y especialmente antes de sus competiciones.

Esta investigación, es la primera en analizar de manera conjunta el efecto agudo de los tipos de entrenamiento de velocidad, fuerza, pliometría y velocidad contra resistencia en la carrera de velocidad y sus fases.

Además, esta investigación está aportando conocimiento nuevo con la incursión en la evaluación de distancias mayores (60, 80 y 100 metros) en atletas competitivos, lo cual permite estudiar las fases de velocidad máxima y de resistencia a la velocidad. Destacan que los estudios previos han estudiado solo la fase de aceleración. 
La información que brinde este estudio, será de utilización práctica y directa para que el atleta y el entrenador puedan individualizar la prescripción del método de entrenamiento agudo para cada atleta.

\section{METODOLOGÍA}

\section{Participantes}

Once velocistas masculinos con una edad promedio de 21.8 años \pm 3.04 , talla de $1.764 \mathrm{~m}$ \pm 0.062 y peso de $69.645 \mathrm{~kg} \pm 4.946$. Los participantes eran atletas de nivel universitario y élites de Costa Rica, con experiencia previa en atletismo. Los participantes llenaron, previo a iniciar la investigación, el consentimiento informado, en el cuál se indicaba con detalle el tratamiento y las pruebas a las que se someterían, así como su derecho a abandonar en el momento que quisieran. El consentimiento informado cumplió con los estándares éticos planteados en la declaración de Helsinki. Cabe destacar, que previo a que los participantes dieran su consentimiento informado, se les explicó de manera verbal y escrita los beneficios y riesgos que conlleva su participación, además, estos contaron con tiempo suficiente para leerlo y realizar las preguntas necesarias.

\section{Instrumentos de medición}

Se utilizaron dos equipos de fotoceldas de las marcas Brower timing system (modelo: CML5MEM; fabricante: Brower Timing System, Draper, Estados Unidos) y Smart speed (modelo: SM-G610F; fabricante: Sport Fusion, Brisbane Australia) para tomar los tiempos totales y parciales de carrera de manera electrónica. Además, se utilizaron un odómetro marca Keson (modelo RR3M; Fabricante: KESON, Estados Unidos) y conos para medir y marcar las distancias a investigar. Cabe destacar que todas las mediciones se llevaron a cabo en el carril seis en la pista de atletismo de la Universidad de Costa Rica, la cual cuenta con las medidas oficiales. Se utilizó una máquina Smith para realizar la prueba de una repetición máxima de media sentadilla al inicio de la investigación, y para el tratamiento de fuerza. Las medidas antropométricas se llevaron a cabo con un tallímetro y báscula marca SECA (modelo 286 fabricante: SECA, Alemania).

\section{Procedimientos}

Para obtener a los participantes para la presente investigación, se contactó tanto a ellos como a sus respectivos entrenadores, explicándoles el objetivo de la investigación y la utilidad que esta representaría para ellos. 
Los participantes asistieron en una primera ocasión, donde se les entregó el consentimiento informado para que lo leyeran con detalle y lo firmaran en caso de estar de acuerdo en participar en la investigación. Posterior a el consentimiento de los participantes, se obtuvieron las mediciones antropométricas básicas (talla y peso), pasando después de estas a la sala de pesas donde se realizó la prueba de una repetición máxima siguiendo un protocolo previamente establecido y utilizado en deportistas (Haff y Triplett, 2015).

Luego del primer día de mediciones generales, se aleatorizó el orden de los tratamientos para todos los participantes, ya que todos debían de realizar todos los tratamientos en días diferentes. Los participantes debían asistir cinco días no consecutivos para realizar las condiciones de fuerza, velocidad, pliometría, velocidad contra resistencia y control.

Para cada tratamiento, se contó con un calentamiento, el cual fue de cinco minutos de carrera moderada más la ejecución de cinco ejercicios técnicos (skipping bajo, medio y alto, talón al glúteo de manera unilateral, se realizaba con la derecha y con la izquierda) y un sprint de 30 metros. Después, los sujetos realizaban el pretest de 100 metros, tenían cinco minutos de descanso e iniciaban el tratamiento asignado para el día. Posterior al tratamiento los sujetos contaban con un descanso de 20 minutos y realizaban el pos test de 100 metros. Se les dio 20 minutos de descanso para igualar los tiempos de espera que los atletas élite deben aguardar en las competencias internacionales entre el calentamiento y la competición.

El test utilizado fue la prueba de 100 metros, la cual se midió como distancia completa y por intervalos de cada 20 metros, con el fin de observar el cambio en la velocidad en las distintas fases de la carrera de velocidad.

El tratamiento de fuerza consistió en la realización de tres series de tres repeticiones de media sentadilla al $90 \%$ de una repetición máxima, realizada en una máquina Smith. Se contó con dos minutos de descanso entre cada serie.

El tratamiento de velocidad trató en seis repeticiones de 30 metros de carrera a máxima intensidad, en las que se contó con 2 minutos de descanso entre cada repetición. Para el tratamiento de velocidad contra resistencia se realizaron las mismas repeticiones de la misma distancia que el tratamiento de velocidad, con el cambio de que los sujetos debían correr arrastrando un trineo con pesas, cuyo peso total fue el equivalente a $15 \%$ de su peso corporal.

Para el tratamiento de pliometría, se realizaron 4 series de 10 repeticiones ( 1 serie de 10 repeticiones por cada ejercicio) de los ejercicios de segundo de triple, saltos con ambos pies sobre vallas $(0.76 \mathrm{~m})$, drop jump $(0.60 \mathrm{~m})$ y segundo de triple otra vez. Al igual que en los tratamientos anteriores, se contó con dos minutos de descanso entre cada serie. 
Finalmente, en la condición control los sujetos debía permanecer sentados en un camerino techado durante los 20 minutos entre el pre y el post test.

\begin{abstract}
Análisis estadísticos
Los datos se analizaron tanto de manera grupal como individual, utilizando estadísticos descriptivos y gráficos para el análisis individual, con el fin de observar si existen respuestas diferentes entre participante.

Por su parte, para el análisis grupal se realizaron dos análisis de varianza (ANOVA), uno de dos vías de medidas repetidas en ambos factores (tratamientos $x$ mediciones), con el objetivo de analizar el efecto de los tratamientos y mediciones en el tiempo total de carrera. El factor tratamientos con cinco niveles (fuerza, velocidad, pliometría, velocidad contra resistencia y control) y el factor mediciones con dos niveles (pre y pos test). El segundo análisis de varianza fue de tres vías de medidas repetidas en los 3 factores (tratamientos $x$ mediciones $x$ intervalos), en el que se analizó el efecto de los tratamientos, los intervalos y las mediciones en el tiempo de carrera. El factor tratamientos con cinco niveles (fuerza, velocidad, pliometría, velocidad contra resistencia y control), el factor mediciones con dos niveles (pre y pos test) y el factor intervalos con 5 niveles $(0-20 \mathrm{~m}, 20-40 \mathrm{~m}, 40-60 \mathrm{~m}, 60-80 \mathrm{~m}$ y $80-100 \mathrm{~m})$.
\end{abstract}

\title{
RESULTADOS \\ En la tabla 2, se presenta la estadística descriptiva de cada pre test y pos test en la distancia total de 100 metros para cada tipo de entrenamiento. Se puede apreciar que solo las condiciones de velocidad y control muestran disminuciones en el tiempo de carrera de pre a pos test, con porcentajes de cambio que representan cambios de 6 y 7 centésimas respectivamente.
}


Tabla 2.

Mediciones de 100 metros pre y pos intervenciones por tipo de entrenamiento. *

\begin{tabular}{lcccc}
\hline Tipo de entrenamiento & Pre \pm DE $(\mathrm{s})$ & Pos \pm DE (s) & $\begin{array}{c}\text { Diferencia } \\
\text { absoluta }(\mathrm{s})\end{array}$ & $\begin{array}{c}\text { Porcentaje de } \\
\text { cambio }\end{array}$ \\
\hline Fuerza & $12.00 \pm 0.62$ & $12.08 \pm 0.67$ & 0.080 & 0.67 \\
Velocidad & $11.88 \pm 0.44$ & $11.82 \pm 0.49$ & -0.060 & -0.51 \\
Pliometría & $11.88 \pm 0.54$ & $11.99 \pm 0.59$ & 0.110 & 0.93 \\
Velocidad contra & & & & \\
resistencia & $11.90 \pm 0.50$ & $11.92 \pm 0.59$ & 0.020 & 0.17 \\
Control & $11.85 \pm 0.54$ & $11.78 \pm 0.50$ & -0.070 & -0.59 \\
\hline
\end{tabular}

Nota: $\mathrm{DE}=$ desviación estándar; $\mathrm{s}=$ segundos; ${ }^{*}=$ Valores de las mediciones pre y pos test son presentados como media y desviación estándar; Nivel de significancia utilizado $=p<0.05$. Fuente: elaboración propia.

Por su parte, no hubo interacción significativa entre los factores mediciones y tipo de entrenamiento (figura 1), lo cual deja claro que no hay cambios en la velocidad de carrera de pre a pos test para ningún tipo de entrenamiento.

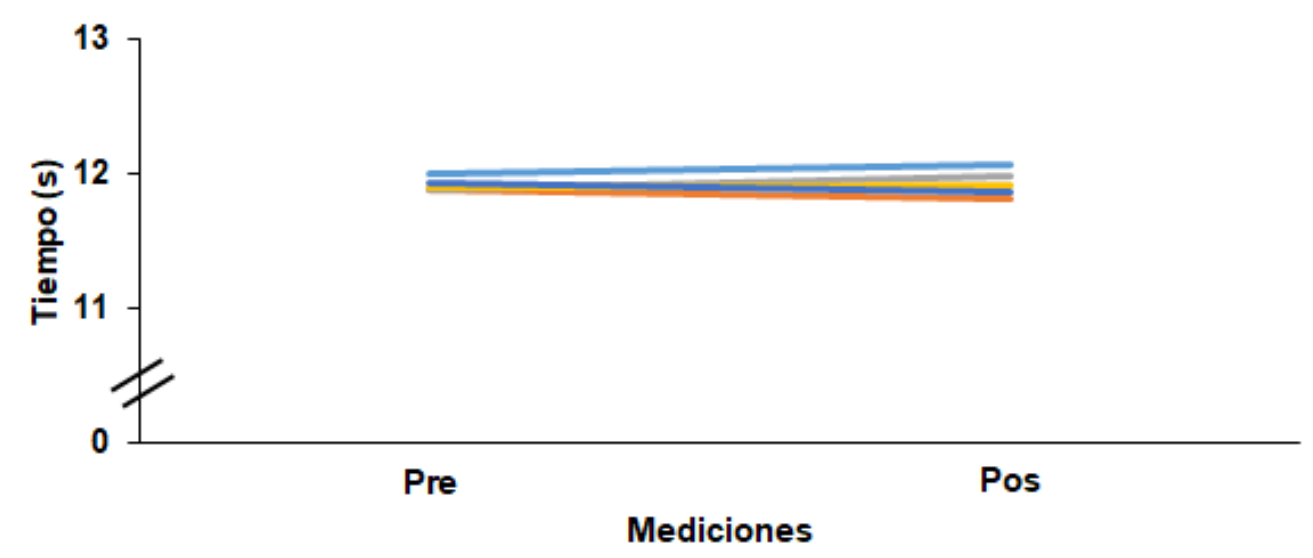

Figura 1. Interacción doble entre los factores mediciones y tratamientos. $\mathrm{F}=1.733 ; p=0.162$. Fuente: elaboración propia.

En la tabla 3 , se presenta la información completa del análisis de varianza de 2 vías de medidas repetidas en ambos factores, donde se observa al igual que en la figura anterior, la 
ausencia de interacción significativa entre los tipos de entrenamiento y las mediciones influenciando la carrera de velocidad.

Tabla 3.

Resumen del análisis de varianza de dos vías de medidas repetidas en ambos factores

\begin{tabular}{lccccc}
\hline Factor & $g l$ & $\begin{array}{c}\text { Suma de } \\
\text { cuadrados }\end{array}$ & Cuadrado medio & $\mathrm{F}$ & $p$ \\
\hline Tratamientos & 4 & 0.007 & 0.007 & 0.231 & 0.641 \\
Mediciones & 1 & 0.655 & 0.164 & 1.31 & 0.283 \\
TxM & 4 & 0.146 & 0.037 & 1.733 & 0.162 \\
\hline
\end{tabular}

Nota: $\mathrm{T}$ = tratamiento; $\mathrm{M}=$ medición; $p=$ significancia; $g l$ = grados de libertad. Nivel de significancia utilizado $=p<0.05$. Fuente: elaboración propia.

También, se muestra la estadística descriptiva de cada pre test y post test por cada intervalo de distancia en cada tipo de entrenamiento (tabla 4), donde los cambios más considerables se pueden observar en el quinto intervalo $(80-100 \mathrm{~m})$ para las condiciones de velocidad y control (7 y 5 centésimas respectivamente). 
Tabla 4.

Mediciones de tiempo de carrera de velocidad pre y pos intervenciones por tipo de entrenamiento y por intervalo de distancia. *

\begin{tabular}{|c|c|c|c|c|c|}
\hline $\begin{array}{c}\text { Tipo de } \\
\text { entrenamiento }\end{array}$ & $\begin{array}{c}\text { Intervalo de } \\
\text { distancia }\end{array}$ & Pre test $\pm \mathrm{DE}(\mathrm{s})$ & $\begin{array}{c}\text { Post test } \pm \\
\text { DE (s) }\end{array}$ & $\begin{array}{c}\text { Diferencia } \\
\text { absoluta (s) }\end{array}$ & $\begin{array}{l}\text { Porcentaje } \\
\text { de cambio }\end{array}$ \\
\hline \multirow{5}{*}{ Fuerza } & $0-20 m$ & $2.98 \pm 0.16$ & $3.02 \pm 0.16$ & 0.040 & 1.34 \\
\hline & $20-40 m$ & $2.21 \pm 0.09$ & $2.25 \pm 0.12$ & 0.040 & 1.81 \\
\hline & $40-60 m$ & $2.17 \pm 0.13$ & $2.20 \pm 0.14$ & 0.030 & 1.38 \\
\hline & $60-80 \mathrm{~m}$ & $2.20 \pm 0.12$ & $2.21 \pm 0.14$ & 0.010 & 0.45 \\
\hline & $80-100 m$ & $2.43 \pm 0.20$ & $2.40 \pm 0.20$ & -0.030 & -1.23 \\
\hline \multirow{5}{*}{ Velocidad } & $0-20 m$ & $3.03 \pm 0.09$ & $3.04 \pm 0.13$ & 0.010 & 0.33 \\
\hline & $20-40 m$ & $2.21 \pm 0.09$ & $2.23 \pm 0.13$ & 0.020 & 0.90 \\
\hline & $40-60 m$ & $2.15 \pm 0.09$ & $2.15 \pm 0.10$ & 0.000 & 0.00 \\
\hline & $60-80 \mathrm{~m}$ & $2.17 \pm 0.09$ & $2.14 \pm 0.10$ & -0.030 & -1.38 \\
\hline & $80-100 m$ & $2.32 \pm 0.18$ & $2.25 \pm 0.17$ & -0.070 & -3.02 \\
\hline \multirow{5}{*}{ Pliometría } & $0-20 m$ & $2.99 \pm 0.13$ & $3.05 \pm 0.16$ & 0.060 & 2.01 \\
\hline & $20-40 m$ & $2.21 \pm 0.10$ & $2.25 \pm 0.11$ & 0.040 & 1.81 \\
\hline & $40-60 m$ & $2.17 \pm 0.11$ & $2.20 \pm 0.10$ & 0.030 & 1.38 \\
\hline & $60-80 \mathrm{~m}$ & $2.20 \pm 0.11$ & $2.20 \pm 0.11$ & 0.000 & 0.00 \\
\hline & $80-100 m$ & $2.31 \pm 0.17$ & $2.30 \pm 0.17$ & -0.010 & -0.43 \\
\hline \multirow{5}{*}{$\begin{array}{l}\text { Velocidad } \\
\text { contra } \\
\text { resistencia }\end{array}$} & $0-20 m$ & $2.96 \pm 0.16$ & $2.96 \pm 0.18$ & 0.000 & 0.00 \\
\hline & $20-40 m$ & $2.23 \pm 0.11$ & $2.25 \pm 0.11$ & 0.020 & 0.90 \\
\hline & $40-60 m$ & $2.16 \pm 0.11$ & $2.16 \pm 0.11$ & 0.000 & 0.00 \\
\hline & $60-80 \mathrm{~m}$ & $2.18 \pm 0.10$ & $2.17 \pm 0.12$ & -0.010 & -0.46 \\
\hline & $80-100 m$ & $2.37 \pm 0.14$ & $2.38 \pm 0.21$ & 0.010 & 0.42 \\
\hline \multirow{5}{*}{ Control } & $0-20 m$ & $3.06 \pm 0.11$ & $3.06 \pm 0.13$ & 0.000 & 0.00 \\
\hline & $20-40 m$ & $2.21 \pm 0.10$ & $2.22 \pm 0.08$ & 0.010 & 0.45 \\
\hline & $40-60 m$ & $2.16 \pm 0.11$ & $2.15 \pm 0.09$ & -0.010 & -0.46 \\
\hline & $60-80 \mathrm{~m}$ & $2.18 \pm 0.13$ & $2.16 \pm 0.10$ & -0.020 & -0.92 \\
\hline & $80-100 m$ & $2.33 \pm 0.17$ & $2.28 \pm 0.15$ & -0.050 & -2.15 \\
\hline
\end{tabular}

Nota: DE = desviación estándar; * = Valores de las mediciones pre y pos test son presentados como media y desviación estándar; $m$ = metros; $s$ = segundos; Nivel de significancia utilizado = $p<0.05$. Fuente: elaboración propia. 
La interacción triple entre los factores mediciones, distancias y tipo de entrenamiento no da significativa (tabla 5). Lo anterior, significa que no hay un cambio en la velocidad de carrera de pre test a post test para ningún tipo de entrenamiento en ningún intervalo de distancia.

Adicionalmente, se presenta un efecto en el factor intervalo, el cual ya se esperaba debido a la diferencia observada entre los tiempos de carrera entre las diferentes fases de la carrera de velocidad (ver figura 2).

Tabla 5.

Tabla resumen del análisis de varianza de tres vías de medidas repetidas en los tres factores

\begin{tabular}{ccccccc}
\hline & $g l$ & $\begin{array}{c}\text { Suma de } \\
\text { cuadrados }\end{array}$ & $\begin{array}{c}\text { Cuadrado } \\
\text { medio }\end{array}$ & $\mathrm{F}$ & $p$ & $\begin{array}{c}\text { Omega } \\
\text { cuadrado }\end{array}$ \\
\hline Tratamientos & 4 & 0.084 & 0.021 & 1.479 & 0.227 & \\
Distancias & 1.375 & 56.444 & 41.044 & 1242.715 & $0.001^{*}$ & $85.64 \%$ \\
Mediciones & 1 & 0.001 & 0.001 & 0.167 & 0.692 & \\
TxD & 16 & 0.377 & 0.024 & 2.253 & $0.006^{*}$ & $0.30 \%$ \\
TxM & 2.284 & 0.029 & 0.013 & 1.733 & 0.196 & \\
DxM & 1.624 & 0.065 & 0.04 & 5.757 & $0.017^{*}$ & $0.08 \%$ \\
TxDxM & 16 & 0.022 & 0.001 & 0.569 & 0.903 &
\end{tabular}

Nota: En los factores de distancias, tratamientos por mediciones y distancias por mediciones, no se asume esfericidad por lo que se utilizan los valores corregidos de Greenhouse-Geisser según lo establecido previamente (O'Donoghue, 2012). $\mathrm{T}$ = tratamiento; $\mathrm{M}$ = medición; $\mathrm{D}=$ distancia; Sig = significancia; $g l=$ grados de libertad. Nivel de significancia utilizado $=p<0.05$. Fuente: elaboración propia. 


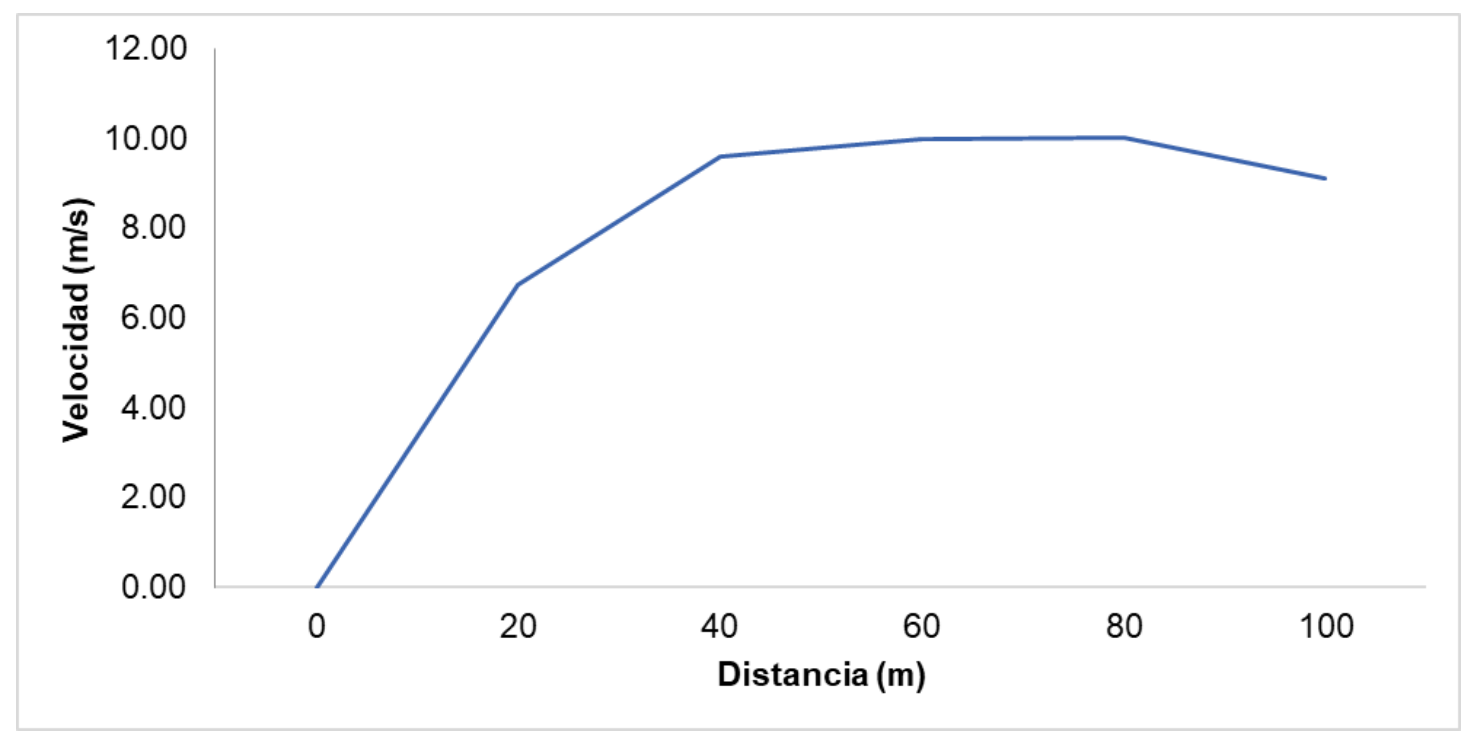

Figura 2. Fases de la velocidad observadas en la carrera de 100 metros planos. Fuente: elaboración propia

Respecto al análisis individual, en la figura 3 se presenta los cambios individuales de pre a post test para el tipo de entrenamiento de velocidad. Se puede apreciar como de manera global no hay efecto de los tipos de entrenamiento en el tiempo de carrera de 100 metros, pero en el análisis individual si se observan cambios (positivos o negativos) en el tiempo de carrera entre los participantes para un mismo tipo de entrenamiento.

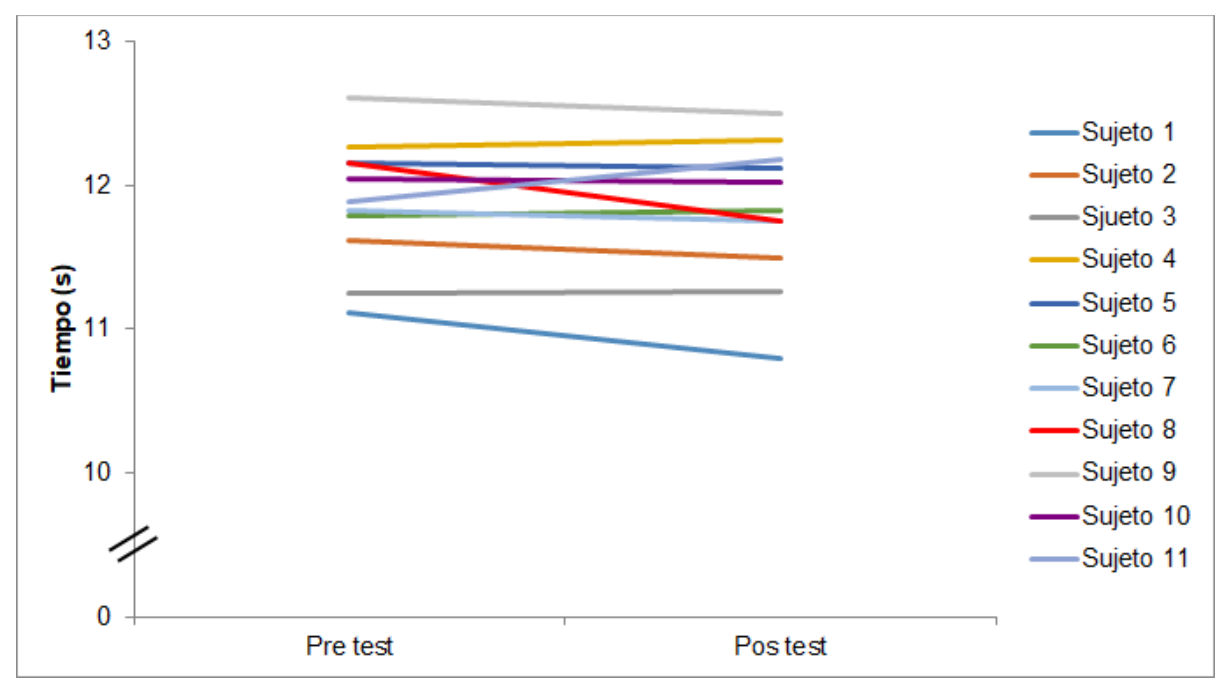

Figura 3. Cambios individuales de pre a post test para el tratamiento de velocidad. Fuente: elaboración propia. 
En la figura 4 se observa los análisis de los cambios entre el pre y el post test en los cinco tratamientos para un participante, donde queda en evidencia una mejora mínima con el entrenamiento de pliometría, dos mejoras muy considerables con los entrenamientos de velocidad y velocidad contra resistencia, y dos empeoramientos de magnitud considerable para el entrenamiento de fuerza y la condición control.

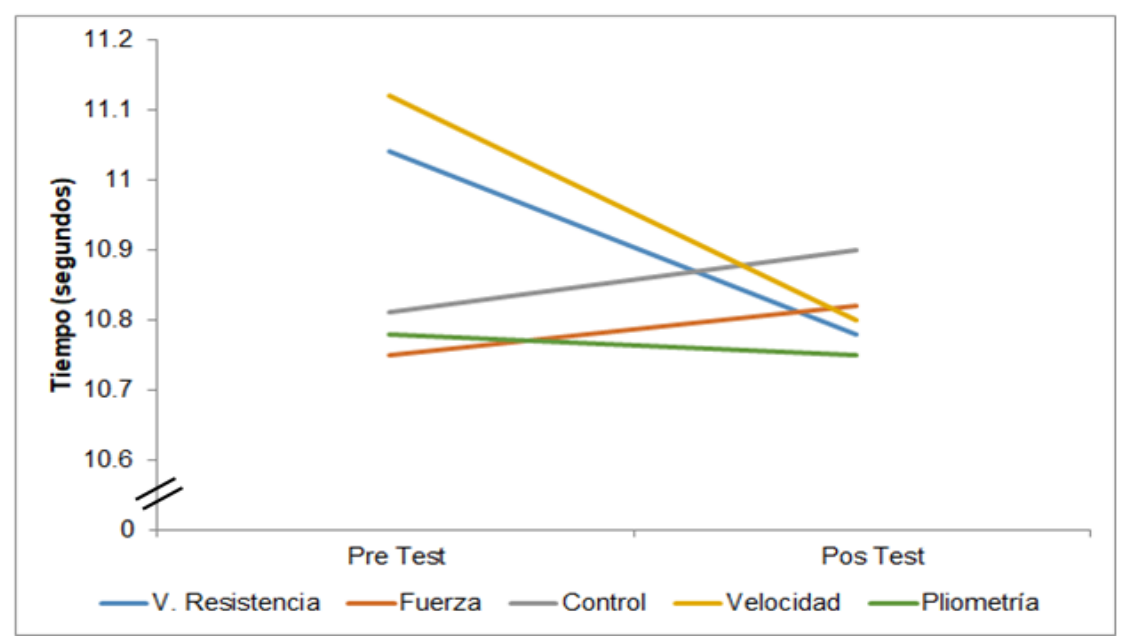

Figura 4. Comparación individual del cambio de pre a pos test para los cinco tratamientos. Nota: V. Resistencia $=$ velocidad contra resistencia. Fuente: elaboración propia.

En la tabla 6 se presenta de forma resumida la cantidad sujetos por tipo de tratamiento que mejoraron, no cambiaron o empeoraron el tiempo de carrera. Se evidencian las diferencias en el tiempo de carrera con distintos tratamientos para cada sujeto, presentándose solo dos sujetos que mejoran con los mismos tratamientos. 
Tabla 6.

Resumen de los cambios individuales experimentados con los cinco tratamientos.

\begin{tabular}{cccccc}
\hline Sujetos & Fuerza & Velocidad & Pliometría & V. Resistencia & Control \\
\hline 1 & - & + & + & $=$ & $=$ \\
2 & - & - & - & - & + \\
3 & + & $=$ & - & + & - \\
4 & - & $=$ & - & - & $=$ \\
5 & - & + & - & - & + \\
6 & - & + & - & + & - \\
7 & - & $=$ & - & + & - \\
8 & $=$ & + & - & - & - \\
9 & - & + & - & - & - \\
10 & + & $=$ & - & + & + \\
11 & - & - & - & - & + \\
\hline Mejoraron & 2 & 5 & 1 & 4 & 4 \\
Sin cambio & 1 & 4 & 1 & 1 & 2 \\
Empeoraron & 8 & 2 & 9 & 6 & 5
\end{tabular}

Nota: Se considera cambio a partir de 0.05 segundos. (+): cambio positivo que implica una disminución del tiempo de carrera; (=): no hay cambio en el tiempo de carrera; (-): cambio negativo que implica un aumento en el tiempo de carrera. Fuente: elaboración propia.

Se observa como el tratamiento de velocidad es con el que más sujetos mejoraron la velocidad de carrera, seguido de los tratamientos de velocidad contra resistencia y control. Por su parte, los tratamientos de fuerza y pliometría fueron con los que más sujetos empeoraron su velocidad de carrera entre pre test y post test.

\section{DISCUSIÓN}

Similar a investigaciones anteriores sobre el efecto agudo de los tipos de entrenamiento de fuerza (Bevan et al., 2010; Crewther et al., 2011; Guggenheimer et al., 2009; Lim y Kong, 2013) y velocidad contra resistencia (Van Den Tillaar et al.,2017; Whelan et al., 2014) en la carrera de velocidad, no se encontró un efecto positivo de estos entrenamientos. Sin embargo, tanto con los tipos de entrenamiento de fuerza (Chatzopoulos et al., 2007; Matthews et al., 2004; Mcbride 
et al., 2005; Rahman, 2007; Seitz et al., 2014; Yetter y Moir, 2008) y velocidad contra resistencia (Smith et al.,2014; Winwood et al., 2016) se han encontrado mejorías significativas.

En el caso de los estudios que evidencian mejoras en la velocidad después de un tratamiento agudo de velocidad contra resistencia, se diferencian de la presente investigación en que las distancias evaluadas para medir velocidad son muy cortas, siendo 15 metros y de 36.5 metros las distancias utilizadas, por lo que estos estudios solo han podido evidenciar mejoras en la fase de aceleración y no en las fases de velocidad máxima y resistencia a la velocidad. Cabe destacar, que al igual que investigaciones anteriores (Van Den Tillaar et al., 2017; Whelan et al., 2014), la investigación presente no encontró mejora en la fase de aceleración con este tratamiento.

Evaluando los tiempos de recuperación entre los tratamientos y el post test, se encuentran estudios que dieron de 4 a 12 minutos con mejora en la velocidad (Smith et al., 2014; Winwood et al., 2016), así como estudios sin mejoras con tiempos de 10 a 12 minutos (Van Den Tillaar et al., 2017; Whelan et al., 2014), por lo que, aunque en la presente investigación este tiempo fue de 20 minutos, no hay evidencia de que tiempos menores sean mejores. Además, uno de los propósitos de esta investigación era dar un tiempo mayor entre el tratamiento y el post test, para simular las situaciones reales de competencias del atletismo de nivel mundial, donde el atleta se ve obligado a esperar 20 minutos en la cámara de llamadas antes de pasar al lugar de competencia.

Las investigaciones del efecto agudo del entrenamiento de fuerza en la carrera de velocidad previamente realizadas, difieren con el presente estudio en que miden la velocidad con distancias más cortas, por ejemplo distancias de 20, 30 y 40 metros, mientras que en el estudio presente se utilizó los 100 metros (y las distancias parciales de 20, 40, 60 y 80 metros), distancia oficial de la prueba de velocidad más importante (Chatzopoulos et al., 2007; Matthews et al., 2004; Mcbride et al., 2005; Rahman, 2007; Seitz et al., 2014; Yetter y Moir, 2008). Lo anterior, es fundamental para poder analizar las fases menos estudiadas de velocidad máxima y resistencia a la velocidad (Haugen et al., 2019).

Otra diferencia importante en la metodología es que en los estudios previos, en los que se encontró mejoría en la velocidad, se utilizó una única distancia para la medición de la velocidad (Chatzopoulos et al., 2007; Matthews et al., 2004; Mcbride et al., 2005; Rahman, 2007; Seitz et al., 2014). En este estudio, además de medir la velocidad en la distancia de 100 metros, se midió la velocidad cada 20 metros, no encontrando efectos de los tipos de entrenamiento en las fases de aceleración, velocidad máxima y resistencia a la velocidad. 
En el caso del efecto agudo del entrenamiento pliométrico en la velocidad, se obtiene que no hay efecto, lo cual es contrario a lo encontrado en investigaciones anteriores (Bomfim-Lima et al., 2011; Turner et al., 2015). En este caso, destacan tres diferencias entre los estudios y la presente investigación, siendo la primera que los tratamientos de pliometría aplicados en estas investigaciones son de menor volumen (10 repeticiones en estudio de Lima et al vs 40 en estudio presente). La segunda diferencia está en que los tiempos de espera entre los tratamientos y el post test son menores al utilizado en la investigación presente, sin embargo, estas mejoras se aumentaban conforme aumentaba el tiempo de recuperación, siendo el tiempo máximo registrado 15 minutos (Bomfim-Lima et al., 2011), resultado contrario al encontrado en el presente estudio donde el tiempo de recuperación fue de 20 minutos y no hubo mejoría en la velocidad. Por último, estos estudios evaluaron la velocidad en distancias totales sin intervalos (Bomfim-Lima et al., 2011) y con un solo intervalo (Turner et al., 2015), razón por lo que solo se pudo comparar los valores de aceleración en el caso de los 10 y 20 metros evaluados por Turner y colaboradores y la velocidad máxima en los 50 metros en el caso de Lima y colaboradores. En ambos estudios se obtuvieron mejoras en el tiempo de carrera, lo cual difiere del presente estudio en el que no se presentaron cambios en la velocidad en ninguna fase de la carrera ni en el tiempo total. También, una diferencia presente solo con la investigación de Turner et al. (2015), es que los sujetos utilizados eran no entrenados, caso contrario al presente estudio en el que se contó con atletas. Podría ser que los estímulos necesarios para provocar cambios en las poblaciones no entrenadas sean menores a los requeridos en atletas. Este comportamiento ya ha sido reportado en el estudio meta-analítico de Barquero y Salazar (2018), donde se encontró que los sujetos no entrenados mejoran más la velocidad respecto a los sujetos entrenados cuando se someten al entrenamiento de velocidad.

Por su parte, el entrenamiento de velocidad no presentó mejoras en la carrera de velocidad. En este caso, la ausencia de un efecto agudo del entrenamiento de velocidad, lleva a plantearse si los calentamientos realizados por los atletas en los que realizan carreras de velocidad son realmente útiles o no conllevan a ninguna diferencia en el rendimiento en la carrera de velocidad y sus fases. Destaca la ausencia de investigaciones de efecto agudo en este tipo de entrenamiento.

En el análisis individual, se observa una mayor cantidad de sujetos que mejoran con el tratamiento de velocidad $(n=5)$, seguido por el tratamiento de velocidad contra resistencia $(n=4)$ y la condición control $(n=4)$. Una posible explicación de este comportamiento, es que los tratamientos de velocidad y velocidad contra resistencia son los más específicos al gesto evaluado, por lo que se estaría observando el principio de especificidad (Bompa y Buzzichelli, 
2015). Esto coincide con la poca cantidad de sujetos que mejoraron con los tratamientos de fuerza y pliometría, donde resalta el hecho de que la mayoría empeoraron (fuerza = 8, pliometría =9).

A pesar de que en el análisis grupal con ningún tratamiento se encontró mejoría significativa en la carrera de velocidad, se discuten los mecanismos explicativos mencionados en la literatura científica, asociados con mejoras en actividades explosivas como la carrera de velocidad. Estos son el mecanismo de potenciación pos-activación (Tillin y Bishop, 2009) y la similitud biomecánica entre los tratamientos y la carrera de velocidad (Mero, Komi y Gregor, 1992).

En este último destacan el entrenamiento de velocidad y velocidad contra resistencia por tener las mismas y muy similares características biomecánicas, mientras que el entrenamiento de pliometría permite mejorar la aplicación de fuerzas en tiempos mínimos de contacto por medio del ciclo de estiramiento-acortamiento, presente también en la carrera de velocidad, y por el aumento del tono muscular previo al contacto con el suelo, debido a un aumento en la velocidad de activación neuromuscular (Mero et al., 1992). Adicionalmente, los cuatro tipos de entrenamiento se caracterizan por incluir ejercicios con cadenas cinemáticas cerradas, donde la similitud está en la aplicación de fuerzas contra el suelo, factor común con la carrera de velocidad (Beattie, Kenny, Lyons y Carson, 2014).

Respecto al mecanismo de potenciación pos-activación, se ha propuesto que este produce mejoras en actividades explosivas después de haberse sometido a un acondicionamiento agudo o calentamiento con contracciones musculares a intensidades máximas o casi máximas (Hodgson, Docherty y Robbins, 2005; Tillin y Bishop, 2009). Estas mejoras están asociadas a tres factores, siendo estos: a) un aumento en la regulación de la cadena liviana de miosina, permitiendo a través de la salida de calcio del retículo sarcoplástico, un cambio en la sensibilidad de la interacción actina-miosina, conllevando este a una modificación estructural de la miosina y por ende a un aumento en la generación de fuerza producto del mecanismo de los puentes cruzados (Tillin y Bishop, 2009), b) un aumento en el reclutamiento de unidades motoras con presencia de fibras musculares de contracción rápida (Tillin y Bishop, 2009), y c) un cambio en el ángulo de penación de las fibras musculares, el cual entre menor sea más favorecerá la transmisión de fuerza hacia el tendón (Fukunaga, Ichinose, Ito, Kawakami y Fukashiro, 1997).

Por su parte, para discutir el análisis individual, se debe contemplar que la carrera de velocidad mejoró en 5 sujetos con el tipo de entrenamiento de velocidad, en 4 sujetos con el entrenamiento de velocidad contra resistencia y la condición control, en 2 sujetos con el entrenamiento de fuerza y solo en 1 sujeto con el entrenamiento de pliometría. Se descarta que las discrepancias en los resultados individuales entre tratamientos se deban a diferencias entre 
las cargas de los protocolos, debido a que todos fueron desarrollados a intensidades máximas o casi máximas, los volúmenes de cada tratamiento fueron similares en tiempos de ejecución (con rangos entre 7 y 12 segundos de ejecución por serie o ejercicios para fuerza y pliometría y por repetición para velocidad y velocidad contra resistencia) y las pausas entre series fueron idénticas.

Además de aplicar protocolos estandarizados, el diseño de este estudio es de medidas repetidas, cuenta con una condición control y el orden las condiciones fue asignado aleatoriamente, por lo que, la aleatorización se encarga de repartir por igual las variables que pueden afectar el desempeño de los sujetos, asegurando, en este caso, que las diferencias observadas entre sujetos en los tratamientos, se deban a una variabilidad inherente de los mismos y no a variables como la fatiga y otras que se puedan pensar como responsables. Agregando, que estas variables están controladas también por la condición control.

En futuras investigaciones, se deben evaluar si los cambios agudos experimentados por cada atleta, con cada tipo de entrenamiento, son consistentes, evaluando en varias oportunidades a los atletas con cada tipo de entrenamiento. Dicha información permitiría evaluar si los efectos agudos observados en este estudio para cada individuo son reales, o si nada más obedecen a la variabilidad del performance de cada participante.

Además, es necesario que se realicen más investigaciones sobre el efecto agudo de los tipos de entrenamiento de velocidad y pliometría en la carrera de velocidad y sus fases, de manera que se puedan establecer cuáles son las características de los tratamientos donde se evidencian cambios.

Por último, la realización de un meta-análisis del efecto agudo de los tipos de entrenamiento investigados es una opción muy válida, en especial para intentar aclarar la controversia existente en los tipos de entrenamiento de fuerza y velocidad contra resistencia.

En conclusión, no hay un efecto agudo de ninguno de los tipos de entrenamiento analizados en la carrera de velocidad y sus fases, cuando se analizan los efectos a nivel grupal en atletas competitivos. Se observan resultados diferentes cuando se hace un análisis individualizado de los efectos de cada tipo de entrenamiento. De lo anterior, resalta la importancia de analizar los resultados de las investigaciones tanto de forma grupal como de manera individual. 


\section{REFERENCIAS}

Barquero, J. F., y Salazar, W. (2018). Meta-análisis del efecto de los tipos de entrenamiento en la carrera de velocidad y sus fases. Presentado en XXIV Simposio Internacional en Ciencias del Deporte, el Ejercicio y la Recreación. Universidad de Costa Rica, San José, Costa Rica. Recuperado de https://bit.ly/2YEWqQm

Barrantes Segura, A., y Aragón Vargas, L. F. (2018). Reiteraciones de 1RM según rango de movimiento y tiempo de descanso entre intentos. Recuperado de: http://hdl.handle.net/10669/75344

Beattie, K., Kenny, I. C., Lyons, M., y Carson, B. P. (2014). The effect of strength training on performance in endurance athletes. Sports Medicine, 44(6), 845-865. doi: https://doi.org/10.1007/s40279-014-0157-y

Bevan, H. R., Cunningham, D. J., Tooley, E. P., Owen, N. J., Cook, C. J., y Kilduff, L. P. (2010). Influence of postactivation potentiation on sprinting performance in professional rugby players. The Journal of Strength \& Conditioning Research, 24(3), 701-705. doi: https://doi.org/10.1519/JSC.0b013e3181c7b68a

Bomfim-Lima, J. C., Marin, D. P., Barquilha, G., Da Silva, L. O., Puggina, E. F., Pithon-Curi, T. C., y Hirabarai, S. M. (2011). Acute Effects of Drop Jump Potentiation Protocol on Sprint and Counter Movement Vertical Jump Performance. Human Movement, 12(4), 324-330. Recuperado de http://psjd.icm.edu.pl/psjd/element/bwmeta1.element.-psjd-doi102478 v10038-011-0036-4

Bompa, T., y Buzzichelli, C. (2015). Periodization Training for Sports (3 $3^{\text {th }}$ ed.). Champaign, Estados Unidos: Human kinetics. Recuperado de https://us. humankinetics.com/products/periodization-training-for-sports-3rd-edition

Centeno, A., y Salazar, W. (2017). Meta-análisis del efecto del entrenamiento con pliométricos, pesas y sprint sobre la velocidad (Tesis de Maestría). Universidad de Costa Rica, San José, Costa Rica.

Cetin, E., Hindistan, I. E., y Ozkaya, Y. G. (2018). Effect of Different Training Methods on Stride Parameters in Speed Maintenance Phase of 100-m Sprint Running. The Journal of Strength \& Conditioning Research, 32(5), 1263-1272. doi: https://doi.org/10.1519/JSC.0000000000001977

Chatzopoulos, D. E., Michailidis, C. J., Giannakos, A. K., Alexiou, K. C., Patikas, D. A., Antonopoulos, C. B., y Kotzamanidis, C. M. (2007). Postactivation potentiation effects after heavy resistance exercise on running speed. Journal of Strength and Conditioning Research, 21(4), 1278-1281. doi: https://doi.org/10.1519/00124278-200711000-00051 
Crewther, B. T., Kilduff, L. P., Cook, C. J., Middleton, M. K., Bunce, P. J., y Yang, G.Z. (2011). The acute potentiating effects of back squats on athlete performance. The Journal of Strength \& Conditioning Research, 25(12), 3319-3325. doi: https://doi.org/10.1519/JSC.0b013e318215f560

Fukunaga, T., Ichinose, Y., Ito, M., Kawakami, Y., y Fukashiro, S. (1997). Determination of fascicle length and pennation in a contracting human muscle in vivo. Journal of Applied Physiology, 82(1), 354-358. doi: https://doi.org/10.1152/jappl.1997.82.1.354

Guggenheimer, J. D., Dickin, D. C., Reyes, G. F., y Dolny, D. G. (2009). The effects of specific preconditioning activities on acute sprint performance. The Journal of Strength \& Conditioning Research, 23(4), 1135-1139. doi: https://doi.org/10.1519/JSC.0b013e318191892e

Haff, G. G., y Triplett, T. (2015). Essentials of Strength Training and Conditioning (4a ed.). Champaign, Estados Unidos: Human Kinetics. Recuperado de https://us.humankinetics.com/products/essentials-of-strength-training-and-conditioning4th-edition-with-web-resource

Haugen, T., Seiler, S., Sandbakk, Ø., y Tønnessen, E. (2019). The Training and Development of Elite Sprint Performance: An Integration of Scientific and Best Practice Literature. Sports Medicine - Open, 5(1). doi: https://doi.org/10.1186/s40798-019-0221-0

Hodgson, M., Docherty, D., y Robbins, D. (2005). Post-Activation Potentiation: Underlying Physiology and Implications for Motor Performance. Sports Medicine, 35(7), 585-595. doi: https://doi.org/10.2165/00007256-200535070-00004

Kale, M., y Acikada, C. (2016). Effects of stride length and frequency training on acceleration kinematic, and jumping performances. Sport Science Review, 25(3-4), 243-260. https://doi.org/10.1515/ssr-2016-0013

Lim, J. J., y Kong, P. W. (2013). Effects of isometric and dynamic postactivation potentiation protocols on maximal sprint performance. The Journal of Strength \& Conditioning Research, 27(10), 2730-2736. doi: https://doi.org/10.1519/JSC.0b013e3182815995

Matthews, M. J., Matthews, H. P., y Snook, B. (2004). The acute effects of a resistance training warmup on sprint performance. Research in Sports Medicine, 12(2), 151-159. doi: https://doi.org/10.1080/15438620490460503

Mcbride, J. M., Nimphius, S., y Erickson, T. M. (2005). The acute effects of heavy-load squats and loaded countermovement jumps on sprint performance. Journal of Strength and Conditioning Research, 19(4), 893-897. doi: https://doi.org/10.1519/00124278-200511000$\underline{00029}$ 
Mero, A., Komi, P. V., y Gregor, R. J. (1992). Biomechanics of sprint running: a review. Sports Medicine, 13(6), 376-392. doi: https://doi.org/10.2165/00007256-199213060-00002

O'Donoghue, P. (2012). Statistics for Sport and Exercise Studies. An introduction. Londres; Nueva York: Routledge. Recuperado de https://www.routledge.com/Statistics-for-Sportand-Exercise-Studies-An-Introduction/ODonoghue/p/book/9780415595575

Rahman, R. (2007). The acute effects of heavy versus light-load squats on sprint performance. Facta Universitatis-Series: Physical Education and Sport, 5(2), 163-169. Recuperado de https://scindeks.ceon.rs/article.aspx?artid=0354-47450702163R

Rumpf, M. C., Cronin, J. B., Mohamad, I. N., Mohamad, S., Oliver, J. L., y Hughes, M. G. (2014). The effect of resisted sprint training on maximum sprint kinetics and kinematics in youth. European Journal of Sport Science, 15(5), 374-381. doi: https://doi.org/10.1080/17461391.2014.955125

Sáez, E., Requena, B., y Cronin, J. B. (2012). The effects of plyometric training on sprint performance: A meta-analysis. The Joumal of Strength \& Conditioning Research, 26(2), 575-584. doi: https://doi.org/10.1519/jsc.0b013e318220fd03

Seitz, L. B., Trajano, G. S., y Haff, G. G. (2014). The Back Squat and the Power Clean: Elicitation of Different Degrees of Potentiation. International Journal of Sports Physiology \& Performance, 9(4), 643-649. doi: https://doi.org/10.1123/ijspp.2013-0358

Slawinski, J., Termoz, N., Rabita, G., Guilhem, G., Dorel, S., Morin, J.-B., y Samozino, P. (2017). How 100-m event analyses improve our understanding of world-class men's and women's sprint performance. Scandinavian Journal of Medicine \& Science in Sports, 27(1), 45-54. doi: https://doi.org/10.1111/sms.12627

Smith, C. E., Hannon, J. C., McGladrey, B., Shultz, B., Eisenman, P., y Lyons, B. (2014). The effects of a postactivation potentiation warm-up on subsequent sprint performance. Human Movement, 15(1), 36-44. doi: https://doi.org/10.2478/humo-2013-0050

Tillin, N. A., y Bishop, D. (2009). Factors modulating post-activation potentiation and its effect on performance of subsequent explosive activities. Sports Medicine, 39(2), 147-166. doi: https://doi.org/10.2165/00007256-200939020-00004

Tønnessen, E., Sylta, Ø., Haugen, T. A., Hem, E., Svendsen, I. S., y Seiler, S. (2014). The road to gold: Training and peaking characteristics in the year prior to a gold medal endurance performance. PloS One, 9(7), e101796. doi: https://doi.org/10.1371/journal.pone.0101796 
Turner, A. P., Bellhouse, S., Kilduff, L. P., y Russell, M. (2015). Postactivation potentiation of sprint acceleration performance using plyometric exercise. The Journal of Strength \& Conditioning Research, 29(2), 343-350. https://doi.org/10.1519/JSC.0000000000000647

Van Den Tillaar, R., Teixeira, A., y Marinho, D. (2017). Acute effect of resisted sprinting upon regular sprint performance. Acta Kinesiologiae Universitatis Tartuensis, 23, 19-33. doi: https://doi.org/10.12697/akut.2017.23.02

Whelan, N., O'regan, C., y Harrison, A. J. (2014). Resisted sprints do not acutely enhance sprinting performance. The Journal of Strength \& Conditioning Research, 28(7), 18581866. doi: https://doi.org/10.1519/JSC.00000000000000357

Winwood, P. W., Posthumus, L. R., Cronin, J. B., y Keogh, J. W. (2016). The acute potentiating effects of heavy sled pulls on sprint performance. The Journal of Strength \& Conditioning Research, 30(5), 1248-1254. doi: https://doi.org/10.1519/JSC.0000000000001227

Yetter, M., y Moir, G. L. (2008). The acute effects of heavy back and front squats on speed during forty-meter sprint trials. The Journal of Strength \& Conditioning Research, 22(1), 159-165. doi: https://doi.org/10.1519/JSC.0b013e31815f958d 